\title{
Lifetime of Wannier-Stark states
}

\author{
M. Glück, A. R. Kolovsky[*] and H. J. Korsch \\ Fachbereich Physik, Universität Kaiserslautern, D-67653 Kaiserslautern, Germany
}

June 14, 1999

\begin{abstract}
The lifetime of a Bloch particle in a homogeneous field is calculated numerically as a function of the field amplitude. A simple model explaining the characteristic features of the field dependence of the lifetime is introduced. The theoretical results are compared with the experimental data of Ref. [1] obtained for cold sodium atoms in an accelerated optical lattice.
\end{abstract}

PACS. 0 3.65.-w, 73.20.Dx

In this Letter we address the question of the lifetime of Wannier states which, by definition, are the metastable states of a system with Hamiltonian

$$
H=p^{2} / 2+V(x)+F x, \quad V(x+2 \pi)=V(x) .
$$

(We use a scaling which leaves only two independent parameters - the scaled static force $F$ and the scaled Planck constant $\hbar^{\prime}$ entering the momentum operator.) For a long time this problem was of considerable theoretical interest in the field of solid state physics [2-4]. Recently it has acquired a new impact due to the experiments with cold neutral atoms in an accelerated standing laser wave [1]. For the first time the tunneling rate was directly measured, thus suggesting a unique opportunity for testing a theory.

We briefly recall the main theoretical predictions. The common approach to the problem, known as LandauZener theory [2], predicts an $1 / F$ dependence for the tunneling rate

$$
\Gamma_{n}(F) \sim F \exp \left(-b_{n} / F\right)
$$

In Eq. (2) the index $n$ refers to the Wannier-Stark states originating from the $n$-th Bloch band and the coefficient $b_{n}$ is proportional to the square of the energy gap between $n$-th and $(n+1)$-th bands. We note that Eq. (2) coincides with an estimate for "incoherent" tunneling through $N \sim 1 / F$ barriers separating the bounded motion of a classical particle in any of the potential wells from the region of unbounded motion. However, as mentioned several times in the literature, in the considered case of a periodic potential the tunneling is not "incoherent". Thus, in its best, Eq. (2) can capture only "an average" dependence on $F$ and large fluctuations of $\Gamma_{n}$ are expected when the amplitude of a static force tends to zero $(N \rightarrow \infty)$ [3]. Although this prediction is out of doubt, the particular dependence of $\Gamma_{n}(F)$ [especially for the important case $V(x)=\cos x]$ has not been studied in any detail.

In this paper we calculate the lifetime of the Wannier states in the potential $V(x)=\cos x$. We restrict ourselves

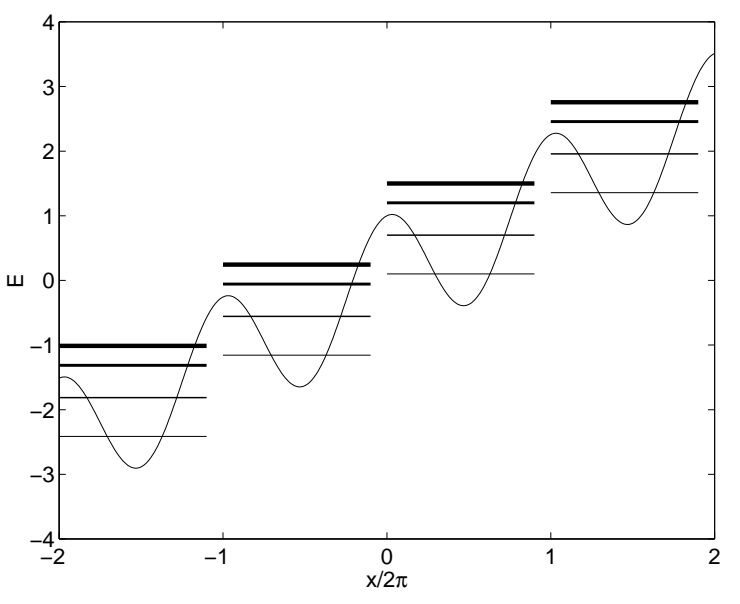

Fig. 1. Schematic drawing of the Wannier-Stark ladder of the resonances. The width of the levels is "proportional" to the resonance width $\Gamma_{n}$ defining the lifetime of the states.

to the case of relatively large values of the scaled Planck constant, where each well of the periodic potential can support no more than one stable state (which essentially simplifies the analysis). In particular we shall be interested in the behavior of the function $\Gamma_{0}(F)$ for $\hbar^{\prime}=1.6711$ used in the laboratory experiment with cold sodium atoms [5]. We explain the peculiarities of $\Gamma_{0}(F)$ observed and continue this function to the region of smaller $F$ unavailable within the present day experimental facilities.

To calculate the lifetime we used the approach developed in our recent papers $[6,7]$. Using this approach one finds the complex energy spectrum of the Wannier states (forming so-called Wannier-Stark ladder of resonances, see Fig. 1) by diagonalizing the unitary matrix $U$ of the system evolution operator over the Bloch period $T_{B}=\hbar^{\prime} / F$ with the nonhermitian resonance-like boundary condition. (We note that all resonances belonging to a particular lad- 

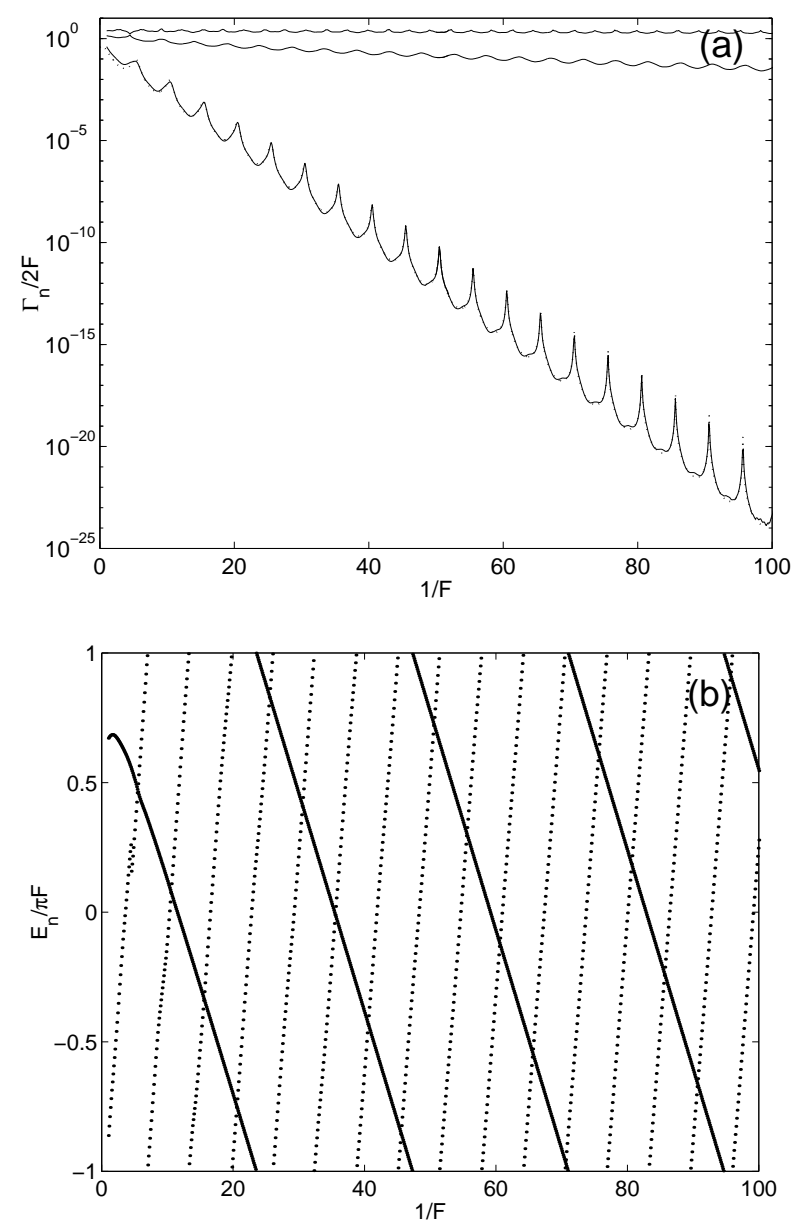

Fig. 2. (a) The width of the first three most stable Wannier resonances as a function of the static field amplitude. [Dotted lines are the approximation of $\Gamma_{0,1}(F)$ by the model (3).] (b) Positions of the ground (solid line) and first excited (dotted line) Wannier resonances reduced to the fundamental energy interval $\Delta E=2 \pi F$. The scaled Planck constant is $\hbar^{\prime}=1.671$.

der correspond to the same eigenvalue of the evolution operator over a Bloch period.) This method proved to be very efficient: it detects not only the states associated with classically bounded motion, but also the metastable states with the energies above the potential barrier.

The results of the numerical calculation are presented in Fig. 2. The dependence of the resonance widths $\Gamma_{n}$ (inverse lifetime) on $F$ is shown in Fig. 2(a) for the first three most stable states. (Note that only the ground state is associated with classically bounded motion.) In addition, Fig. 2(b) shows the positions of the first two Wannier levels reduced to the fundamental energy interval $-\pi F<E_{n}<\pi F$. The following conclusions can be drawn from the figure. (i) Equation (2) captures correctly the average decrease of the tunneling rate as $F$ tends to zero. (ii) Tunneling is enhanced when the different Wannier ladders cross. (iii) The crossings of the levels greatly affect the decay rate of more stable state and almost does not affect less stable state. (iv) Along with the level crossings there are also quasicrossings (see discontinuity of $E_{1}$ around $1 / F=4)$. In this case the Wannier states change their stability, i.e. the second excited state [not shown in Fig. 2(b)] becomes more stable than the first one.

We shall show now that the effect of the enhanced tunneling due to the level crossings can be well mimicked by a simple two-state model. In this model we assign zero width $\bar{\Gamma}_{0}=0$ to the ground level, a finite width $\bar{\Gamma}_{1}=\bar{\Gamma}_{1}(F)=$ $a_{1} F \exp \left(-b_{1} / F\right)$ to the next level [see Eq. (2)], and explicitly introduce a coupling between the states. Then the ground Wannier resonance is given by the largest (by absolute value) eigenvalue $\lambda_{0}=\exp \left(-i\left[E_{0}-i \Gamma_{0} / 2\right] / F\right)$ of the $2 \times 2$ matrix

$$
\begin{gathered}
U=W \bar{U}, \\
\bar{U}_{m, n}=\delta_{m, n} \exp \left(-\frac{i}{F}\left[\bar{E}_{n}(F)-i \frac{\bar{\Gamma}_{n}(F)}{2}\right]\right), \\
W=\exp \left\{-i \epsilon(F)\left(\begin{array}{ll}
0 & 1 \\
1 & 0
\end{array}\right)\right\}, \quad \epsilon(F)=a \exp (-b / F),
\end{gathered}
$$

In Eq. (4) $\bar{E}_{n}(F) \approx E_{n}^{0}+\pi F(n=0,1)$ are the level positions, and the coefficients $a$ and $b$ in Eq. (5) are the adjusted parameters. Because of the coupling, the ground level now gains a finite width $\Gamma_{0}=\Gamma_{0}(F)$ which depends on the relative position of the levels inside the fundamental energy interval. As an example, the dotted line in Fig. 2(a) shows the obtained approximate dependence $\Gamma_{0}(F)$. A good agreement with the exact result is seen. (We also note that the approximation can be improved by using a three-level model [7].)

Furthermore the model (3) yields an analytical expression for $\Gamma_{0}(F)$. In fact, in the considered case of a $2 \times 2$ matrix $U$ one easily solves the eigenvalue problem, which gives

$$
\lambda_{0}=\cos \epsilon\left(\frac{\bar{\lambda}_{0}+\bar{\lambda}_{1}}{2}\right)+\left[\cos ^{2} \epsilon\left(\frac{\bar{\lambda}_{0}+\bar{\lambda}_{1}}{2}\right)^{2}-\bar{\lambda}_{0} \bar{\lambda}\right]^{1 / 2} .
$$

In Eq. (6) we use the short notation $\bar{\lambda}_{n}=\exp \left(-i\left[\bar{E}_{n}-\right.\right.$ $\left.\left.i \bar{\Gamma}_{n} / 2\right] / F\right)$ and omit the argument $F$ of these functions and the function $\epsilon(F)$. Because of $\epsilon \ll 1$ we can simplify Eq. (6) to

$$
\lambda_{0}=\bar{\lambda}_{0}\left[1-\frac{\epsilon^{2}}{2}\left(\frac{\bar{\lambda}_{0}+\bar{\lambda}_{1}}{\bar{\lambda}_{0}-\bar{\lambda}_{1}}\right)\right] .
$$

Then, bearing in mind that $\Gamma_{n}=-2 F \operatorname{Re}\left[\ln \lambda_{n}\right]$, we obtain the equation for the resonance width $\Gamma_{0}$ [8]

$$
\frac{\Gamma_{0}}{F}=\epsilon^{2} \operatorname{Re}\left[\frac{\bar{\lambda}_{0}+\bar{\lambda}_{1}}{\bar{\lambda}_{0}-\bar{\lambda}_{1}}\right] .
$$

Finally, considering the nearest points of local maximum $\Gamma_{0}^{\max }$ and minimum $\Gamma_{0}^{\min }$ (which obviously correspond to the level crossing $\arg \left[\ln \bar{\lambda}_{0}\right]=\arg \left[\ln \bar{\lambda}_{1}\right]$ and the largest distance between the Wannier levels $\arg \left[\ln \bar{\lambda}_{0}\right]=\arg \left[\ln \bar{\lambda}_{1}\right]$ $+\pi$, respectively, see Fig. 2), we obtain a relation which does not contain the adjusted parameters

$$
\frac{\Gamma_{0}^{\max }}{\Gamma_{0}^{\min }} \approx\left(\frac{2 F}{\bar{\Gamma}_{1}(F)}\right)^{2} .
$$




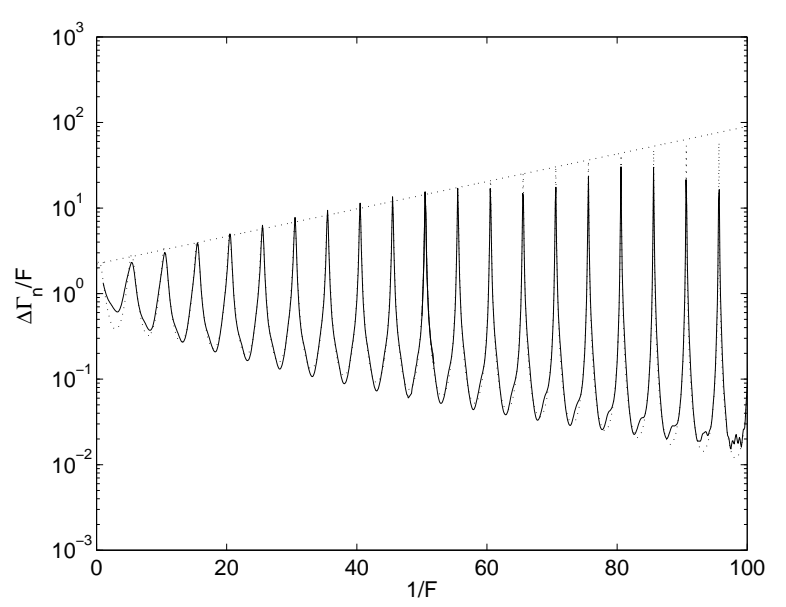

Fig. 3. The fluctuation $\Delta \Gamma_{0}(F)$ of the decay rate of the ground Wannier states as a function of $F$. Solid line is the exact result, dotted line is the approximation by Eq. (7) with $\epsilon(F)=a \exp (-b / F), a=1, b=0.253$. The dotted straight line corresponds to Eq. (9) where $\bar{\Gamma}_{1} / F$ is obtained by fitting the middle curve in Fig. 2(a) by a straight line.

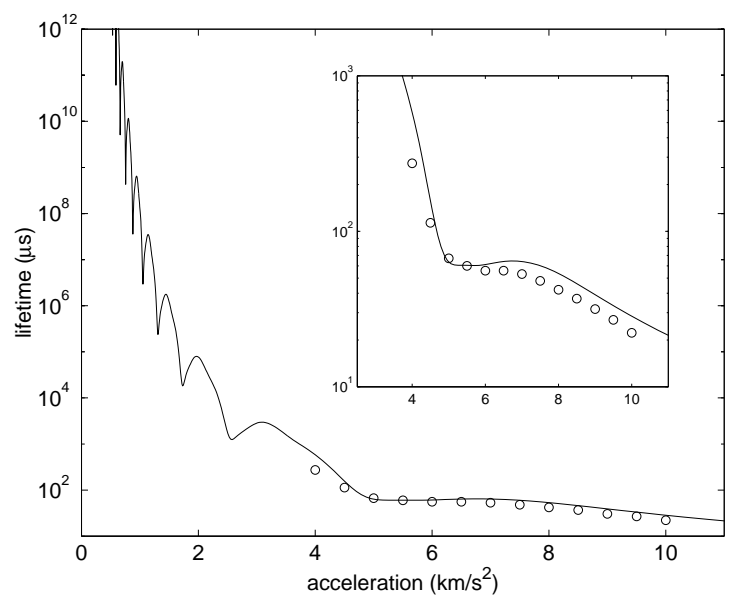

Fig. 4. Lifetime of the ground Wannier state. Solid line is the theoretical result of Fig. 2(a), circles are the experimental data of Ref. [1]. The insert blows up the interval $4000 \mathrm{~m} / \mathrm{s}^{2}<a<$ $10000 \mathrm{~m} / \mathrm{s}^{2}$ considered in the cited experiment.

Equation (9) has a simple physical meaning: the characteristic amplitude for fluctuation of $\Gamma_{n}(F)$ is defined by the average tunneling rate $\bar{\Gamma}_{n+1}(F)$ of the next excited state (see Fig. 3). A justification of this statement in the case of smaller $\hbar^{\prime}$, where a potential well supports many narrow resonances, will be the subject of a separate paper [7].

In this paragraph we compare the numerical data presented with the experimental data of Ref. [1]. In the cited experiment the sodium atoms in the optical lattice of a $\lambda=589 \mathrm{~nm}$ laser were subject to the inertial force due to acceleration of the standing wave. The acceleration was varied in the interval $3000 m / s^{2}<a<10000 m / s^{2}$ which corresponds to the scaled static force $0.113<F<0.373$.
The solid line in Fig. 4 reproduces the theoretical curve of Fig. 2(a) plotted in the initial units. The circles are the measured tunneling rate. It is seen that the experimental data follows the theoretical prediction. (According to Ref. [1] we attribute the deviation to ten-percent uncertainty in the depth of the potential.) In particular we note a drop of the curve at $a=5000 \mathrm{~m} / \mathrm{s}^{2}$ which, as it was explained above, is due to the crossing of the ground and first excited Wannier levels in the neighboring wells.

To conclude, we have numerically determined the lifetime of a Bloch particle in the cosine potential subject to a homogeneous static field. It was confirmed that the lifetime as a function of the static field amplitude follows in average the Landau-Zener formula. However, sharp peaks are superimposed on this smooth dependence. These peaks of enhanced tunneling are due to the crossing of the Wannier ladders. The effect of the level crossing is shown to be well captured by a simple two-level model introduced in the paper. We also would like to note that our approach of calculating the tunneling rate uses the Wannier resonances and does not require information about the dispersion relation for the Bloch bands. This differs it from the complementary "Bloch -band" approach of the recent paper [9].

It is a pleasure to thank J. E. Avron and M. G. Raizen for discussions. This research has been supported by the Deutsche Forschungsgemeinschaft (SPP 470 "Zeitabhängige Phänomene und Methoden in Quantensystemen der Physik und Chemie").

\section{References}

[*] Also at L. V. Kirensky Institute of Physics, 660036 Krasnoyarsk, Russia.

1. C. F. Bharucha, K. W. Madison, P. R. Morrow, S. R. Wilkinson, Bala Sundaram, and M. G. Raizen, Phys. Rev. A 55, R857 (1997).

2. G. Zener, Proc. R. Soc. London, Ser. A 137, 696 (1932); L. D. Landau, Phys. Z. Sov. 1, 46 (1932).

3. J. E. Avron, Ann. Phys. (N.Y.) 143, 33 (1982).

4. Interband Tunneling in Tunneling Phenomena in Solids, edited by E. Burstein and S. Lundqvist (Plenum Press, New York, 1969); Jun He and G. J. Iafrate, Phys. Rev. B 50, 7553 (1994); V. Grecchi and A. Sacchetti, Phys. Rev. Lett. 78, 4474 (1997). See also references in the review articles: G. Nenciu, Rev. Mod. Phys. 63, 91 (1991); E.E. Mendez and G. Bastard, Phys. Today 46, No. 6, 34 (1993); F. Rossi, Semicond. Sci. Technol. 13, 147 (1998); K. Leo, Semicond. Sci. Technol. 13, 249 (1998).

5. For neutral atoms in the optical lattice [the Hamiltonian $\left.H=p^{2} / 2 M+V_{0} \cos \left(2 k_{L} x\right)+M a x\right]$ an appropriate rescaling of the coordinate and time is $x \rightarrow x / 2 k_{L}$ and $t \rightarrow \Omega_{p h} t$ where $\Omega_{p h}$ is the frequency of small atomic oscillations near the bottoms of the cosine potential. Then the value of the scaled Planck constant is given by $\hbar^{\prime}=\left(8 \hbar \omega_{r e c} / V_{0}\right)^{1 / 2}$ ( $\omega_{\text {rec }}=\hbar k_{L}^{2} / 2 M$ is the recoil frequency), the scaled static force is $F=M a k_{L}^{-1} / 2 V_{0}$, and $V(x)=\cos x$ in the dimensionless Hamiltonian (1). In the experiment [1] with sodium atoms we have $\omega_{r e c} / 2 \pi=25.14 k H z$ and $V_{0} / h=$ $72 k H z$. 
6. M. Glück, A. R. Kolovsky, H. J. Korsch, and N. Moiseyev, EPJ D4, 239 (1998); M. Glück, A. R. Kolovsky, H. J. Korsch, Phys. Rev. Lett. 82, 1534 (1999); Phys. Rev. E (1999), in press.

7. M. Glück, A. R. Kolovsky, H. J. Korsch, Resonant tunneling of the Wannier-Stark-States (unpublished)

8. Equation (8) was actually used to determine the adjusted parameters $a$ and $b$ in Eq. (5). The average resonance width $\bar{\Gamma}_{1}(F)$ and the level positions $\bar{E}_{0,1}(F)$ were extracted from Fig. 2 by approximating the corresponding curves by straight lines. Alternatively, these functions can be calculated by using semiclassical techniques.

9. Qian Niu and M. G. Raizen, Phys. Rev. Lett. 80, 3491 (1998); S. R. Wilkinson, C. F. Bharucha, K. W. Madison, Qian Niu, and M. G. Raizen, Phys. Rev. Lett. 76, 4512 (1996); Qian Niu, Xian-Geng Zhao, G. A. Georgakis, and M. G. Raizen, Phys. Rev. Lett. 76, 4504 (1996); 